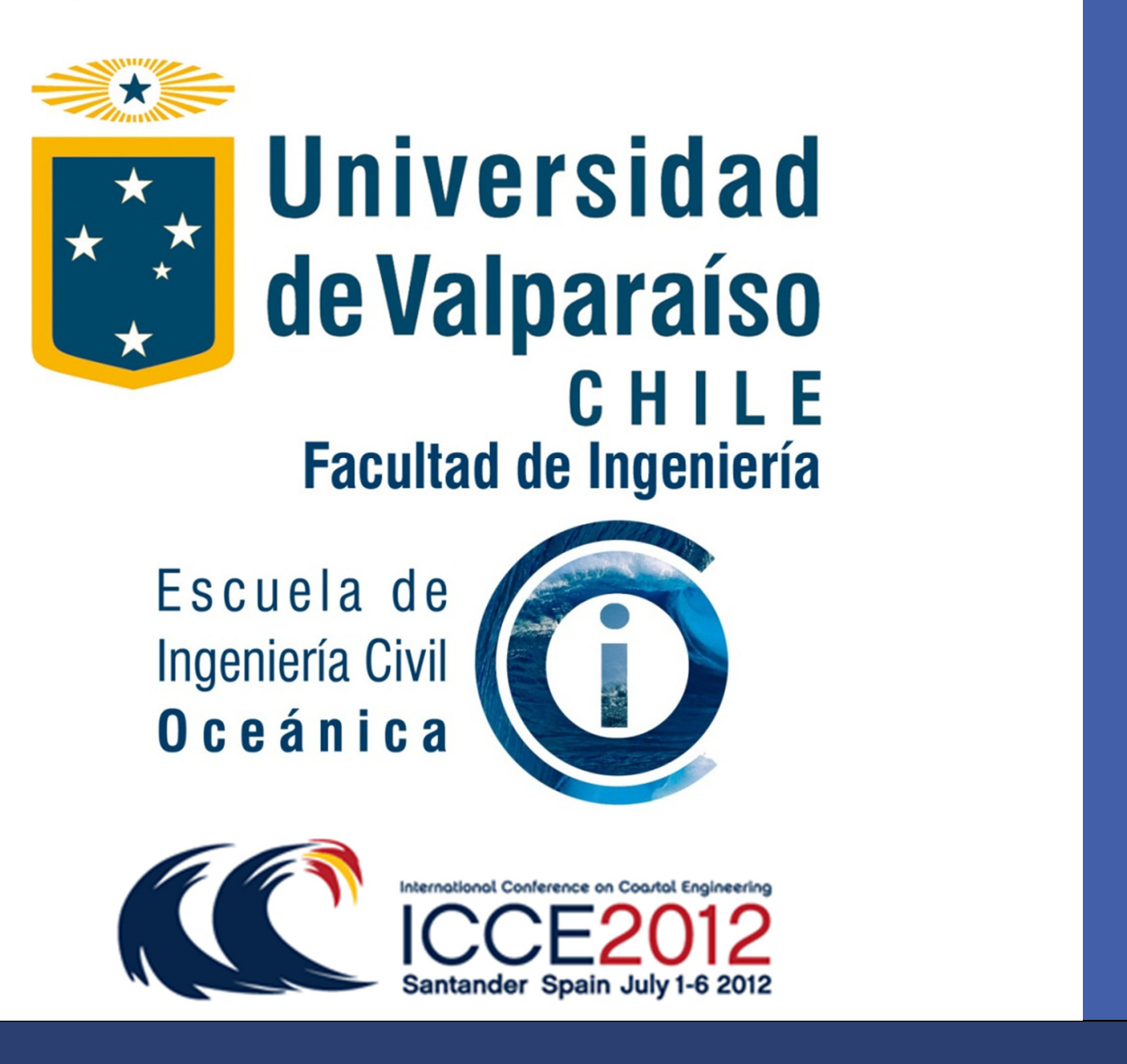

\title{
When do Waves become Turbulent?
}

\author{
José Beyá ${ }^{1}$, William Peirson², Michael Banner ${ }^{3}$ \\ ${ }^{1}$ Ocean Engineering, Facultad de Ingeniería, Universidad de Valparaíso, Chile, jose.beya@uv.cl \\ ${ }^{2}$ Water Research Laboratory, University of New South Wales, Australia, bill.peirson@wrl.unsw.edu.au \\ ${ }^{3}$ School of Mathematics, University of New South Wales, Australia, m.banner@unsw.edu.au
}

\section{INTRODUCTION}

Babanin and Haus (2009) recently presented evidence of high levels of turbulence induced by steep non-breaking waves. They proposed a Reynolds-like threshold wave parameter $\left(a^{2} \omega / v=3000\right.$, a wave amplitude, $\omega$ wave angular frequency, $v$ water viscosity) for the spontaneous occurrence of turbulence beneath surface waves. This is contrary to conventional engineering understanding and the widespread use of irrotational wave theories based on classical experimental validation.

Many laboratory wave experiments were carried out in the early 1960's (e.g. Wiegel, 1964). In those experiments no evidence of turbulence was reported and steep waves behaved as predicted by the high order irrotational wave theories within the accuracy of the theories and experimental techniques at the time.

The spontaneous generation of turbulence under waves can have serious consequences for wave modelling, where the irrotational flow assumption has secured its place in engineering design.

This contribution describes unique flow visualisation experiments for large scale steep non-breaking waves using conventional dye techniques in the wave boundary layer extending above the wave trough level.

\section{EXPERIMENTAL SETUP}

A vertical dye line was created on a still clean water tank of $0.6 \mathrm{~m}$ width (Figure 1 ) Tests cases consisted of $1.5 \mathrm{~Hz}$ deep water monochromatic wave trains amplitudes from $187 \mathrm{~mm}$ to $269 \mathrm{~mm}\left(a^{2} \omega / v\right.$ from 3000 to 7000 , Table 1$)$. Above this amplitude value the first larger wave of the train broke and generated turbulence from the surface diffusing into the water column (Figure 2 ). A video camera mounted on the side of the tank at $5.25 \mathrm{~m}$ from the flexible plate wave generator, recorded the evolution of the dye line during the passage of the wave trains up to $16 T(T=2 \pi / \omega)$ from the time the initiation of motion at the measurement point. Longer lapses were not possible because the stretching of the dye line caused by the Stokes drift made its detection very difficult. Image processing tools were used to rectify and digitise the position and width of the lines.

Table 1 - Test cases of monochromatic wave trains generated from a still water start condition. $\left(\omega=9.42 \mathrm{rads}^{-1}, k_{0}=\omega^{2} / \mathrm{g}=9.05 \mathrm{radm}^{-1}, c_{0}=\omega / k_{0}=1.041 \mathrm{~ms}^{-1}, d=0.405 \mathrm{~m}\right.$ water depth)

\begin{tabular}{|c|c|c|c|}
\hline Case & 1 & 2 & 3 \\
\hline Half wave height, $\mathrm{H} / 2(\mathrm{~mm})$ & 18.7 & 22.7 & 26.9 \\
\hline Wave steepness, $(\mathrm{kH} / 2)$ & 0.17 & 0.21 & 0.24 \\
\hline$a^{2} \omega / v$ & 3295 & 4856 & 6819 \\
\hline Wave number (Fifth order), $k\left(\mathrm{radm}^{-1}\right)$ & 8.88 & 8.91 & 8.94 \\
\hline Wave speed (Fifth order), $c\left(\mathrm{~ms}^{-1}\right)$ & 1.032 & 1.029 & 1.025 \\
\hline
\end{tabular}

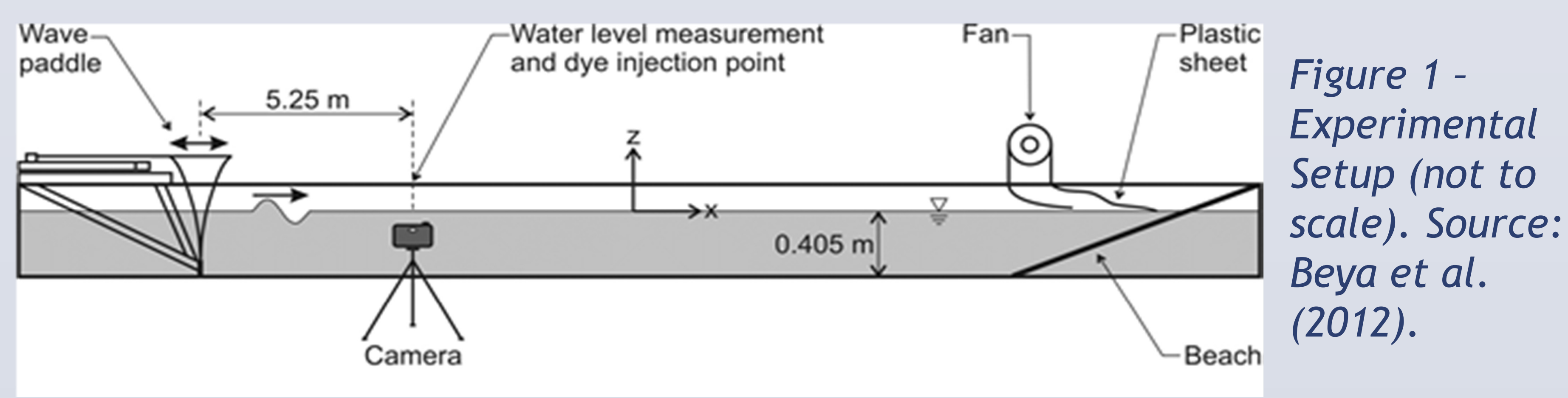

\section{EVIDENCE OF NON TURBULENT FLOW UNDER WAVE TROUGHS}

No evidence of turbulent mixing was observed during any of the experiments (Figure 3 ). The width of the dye lines was measured and no significant widening was detected (Figure 4), in fact, the thinning and stretching caused by the Stokes drift decreased line colour intensity. It was noticed that the dye line did not widen faster than it would due to molecular diffusion. The only evidence of turbulent flow resulted from the breaking of the first larger wave of the train when $a^{2} \omega / v$ was greater than 7000 (Figure 2).

Figure 2 - Photograph showing the breaking of the initial largest wave for a monochromatic wave train $\left(a^{2} \omega / v>7000\right)$ propagating on still water. The image was taken from the tank side wall below the water surface near the tank floor and looking up towards the surface in an angle of approximately $60^{\circ}$. Capillary ripples triggered by side walls can also be observed at the water surface.

\section{COMPARISON WITH IRROTATIONAL NON-LINEAR WAVE THEORIES}

Dye line position measurements were processed to obtain particle orbital motion, orbital velocities and mean velocities which were successfully compared to theoretical predictions of fifth order Stokes waves (Fenton, 1985) and the Stokes drift (Figure 5).

Figure 3 - Dye line under Steep Non-

Breaking

Monochromatic Waves (Case 1). Scales in millimetres. Dotted and dashed lines are the estimated surface elevation and trough level. The dye plume near the surface is from previous tests.
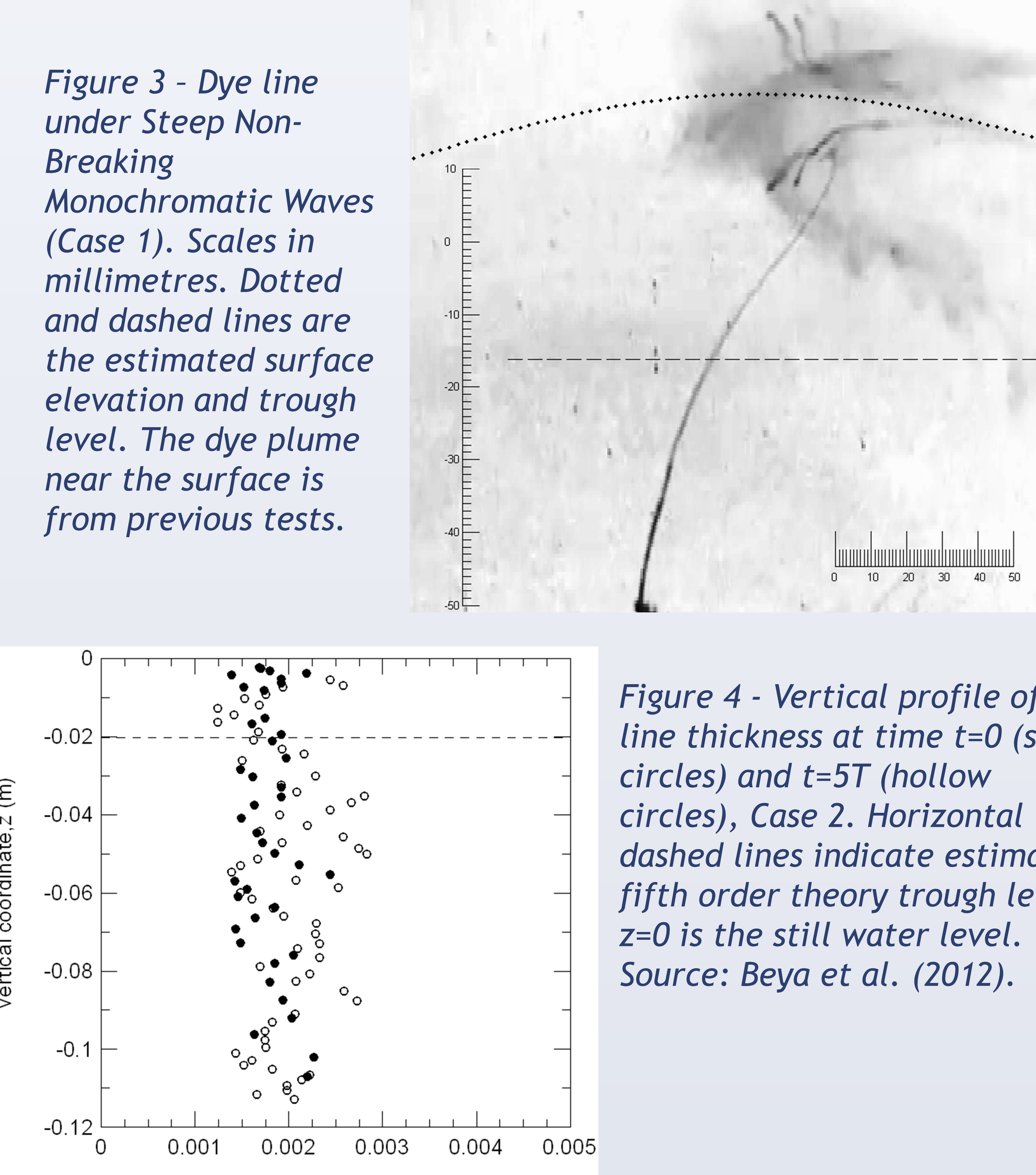

Figure 4 - Vertical profile of dye line thickness at time $t=0$ (solid circles) and $t=5 T$ (hollow circles), Case 2. Horizontal dashed lines indicate estimated fifth order theory trough levels. $\mathrm{z}=0$ is the still water level. Source: Beya et al. (2012).

Figure 5 - Measured particle trajectory, $z=-0.02 m$, Case 3 (circles) from the dye visualization experiment and fifth order theory (solid line). Arrows indicate measured total particle displacement over one wave period where the mean velocity at the average $\mathrm{z}$ is estimated as $\delta / T$. Note the significant difference in position between the theoretical and measured Stokes drift after $2 \frac{1}{4}$ wave cycles.

\section{CONCLUSIONS}

Careful dye measurements showed no evidence of the onset of turbulent behaviour for waves up to $a^{2} \omega / v=7000$ contrary to the conclusions of Babanin and Haus (2009). There is presently no evidence that water waves become spontaneously turbulent up to $a^{2} \omega / v=7000$ except within the bed boundary layers, under wind forcing or at breaking.

Excellent agreement was found with higher order irrotational theories. Orbital velocities, Stokes drift and Stokes coefficients were measured and compared with theoretical values, suggesting that conventional theories underestimate unforced monochromatic wave non-linearity, although the corrections remain small.

\section{REFERENCES}

Babanin, Haus (2009): On the existence of water turbulence induced by non-breaking surface waves, J. Phys. Oceanogr., Notes and Correspondence, 39:2675-2679.

Beya (2011): On the interaction of ocean waves and turbulence, thesis dissertation, UNSW.

Beya, Peirson, Banner (2012): Turbulence beneath finite amplitude water waves, Exp. Fluids, DOI 10.1007/s00348-011-1254-4

Fenton (1985): A fifth-order Stokes theory for steady waves, J. of Waterway, Port, Coastal and Ocean Engineering, 111(2):216-234

Wiegel (1964): Oceanographical Engineering, Prentice-Hall International in Theoretical and Applied Mechanics, N. M. Newmark editor.

\section{ACKOWLEDGEMENTS}

CONICYT - BECAS CHILE.

Ocean Engineering and Faculty of Engineering, Universidad de Valparaíso.

Water Research Laboratory, University of New South Wales. 